\title{
Development of a Wearable Mobile Electrocardiogram Monitoring System by Using Novel Dry Foam Electrodes
}

\author{
Kevin C. Tseng, Member, IEEE, Bor-Shyh Lin, Member, IEEE, Lun-De Liao, Member, IEEE, \\ Yu-Te Wang, and Yu-Lin Wang
}

\begin{abstract}
The development of many telemedicine systems focus on the utility of communication techniques. However, the comfort of long-term Electrocardiogram (ECG) monitoring in daily life is necessary and is also an important unresolved issue. A wearable mobile electrocardiogram monitoring system (WMEMS) is proposed to monitor ECG in this study. It mainly consists of a wearable ECG acquisition device, a mobile phone platform, and a healthcare server. A novel dry foam electrode is also designed and embedded in the wearable ECG acquisition device. The novel dry foam electrode can provide good conductivity to acquire ECG effectively without conduction gels. By using the adapting property of the dry foam electrode, the skin-electrode impedance and the motion artifacts under movement can also be reduced by adapting to irregular skin surface. Moreover, by using the mobile phone as the system platform, the proposed WMEMS can monitor the user's ECG state continuously and anywhere in the globe if they are under the coverage of Global System for Mobile communications (GSM) cellular network. Finally, WMEMS is tested for detecting atrial fibrillation in the hospital. It really provides a good performance and is a good prototype for ECG telemedicine applications.
\end{abstract}

Index Terms-Atrial fibrillation, dry foam electrode, electrocardiogram, telemedicine systems.

\section{INTRODUCTION}

C ARDIOVASCULAR disease (CVD) contains many diseases and conditions of the heart and blood vessels, and it is also one of the main death causes across most of the countries in the world. The World Health Organization estimated that the deaths of almost 17 million people around the globe each year result from CVDs, and about 20 million

Manuscript received May 29, 2010; revised March 25, 2013; accepted March 27, 2013. Date of publication August 7, 2013; date of current version August 21,2014 . This work was supported in part by the National Science Council of R.O.C. for the support of the research through contracts in NSC 100-2221E-009-007 and NSC 101-2221-E-009-039.

K. C. Tseng is with the Department of Industrial Design, Chang-Gung University, Taoyuan, Taiwan.

B.-S. Lin and L.-D. Liao are with the Institute of Imaging and Biomedical Photonics, and Biomedical Electronics Translational Research Center, National Chiao Tung University, Hsinchu, Taiwan (e-mail: borshyhlin@gmail.com).

Y.-T. Wang is with the Swartz Center for Computational Neuroscience, University of California San Diego San Diego, CA USA.

Y.-L. Wang is with the Department of Rehabilitation, Chi Mei Medical Center, Tainan, Taiwan.

Color versions of one or more of the figures in this paper are available online at http://ieeexplore.iee.org.

Digital Object Identifier 10.1109/JSYST.2013.2260620 people encounter the risk of sudden heart failure [1]. However, some of these lives may be saved if prompt emergency care and cardiac surgery can be provided within golden hour. Therefore, real-time electrocardiogram (ECG) monitoring is useful and important for people with CVD.

Recently, many telemedicine systems were developed by integrated with communication technology to enable people to monitor their cardiac health from anywhere at any time [2]-[9]. Rasid and Woodward [8] designed a Bluetooth telemedicine processor to acquire and upload physiological information to a medical healthcare institute via general packet radio service (GPRS) mobile network. Lee et al. use a mobile phone to develop a role-based intelligent mobile care system with alert mechanism to recognize abnormal condition and send an emergency alert message to the healthcare center and physicians [9].

The development of the above mentioned telemedicine systems mainly focuses on the utility of communication techniques. However, in fact, the comfort of monitoring ECG state in daily life is also a necessary and important unresolved issue. As have been highlighted by systems scientists [10]-[13], there are a lot of complexities surrounding this healthcare issue, in particular in emergency situations. Hence, it needs to pay more attentions on designing a useful and comfortable ECG monitoring system. In general, the conventional wet electrodes have been widely used in clinical and applied for the above telemedicine systems. However, the major drawback is that conduction gel used in the wet electrode inevitably leaves its residues on the skin, and this causes the discomfort in use easily. The gel-coated sponge trends to be drying, and this also results in the motion artifact is easily induced by friction and slipping of the electrode. Therefore, the wet electrode is not suitable for long-term ECG monitoring.

In order to overcome the above issues, micro-electromechanical-systems (MEMS) ECG electrodes have been developed and designed in previous studies [14]-[17]. Even though positive results of MEMS electrodes have been published, most of them still have some drawbacks, such as attendant pains when MEMS electrode penetrates into the skin, fragility during the penetration, and high cost for the manufacture procedure of MEMS electrodes. In particular, the fragility of MEMS electrodes may cause damage to the skin over long-term monitoring. 
In order to develop an appropriate dry electrode applied for wearable ECG device, the future design of an effective dry ECG electrode will trend to satisfy several following criteria: 1) noninvasive; 2) no conduction gel used; 3) good ECG signal quality; 4) disposable and low-cost; and 5) good mechanical stability upon attachment on the skin. In this study, a wearable mobile electrocardiogram monitoring system (WMEMS) was proposed to monitor ECG signal in daily life. WMEMS mainly consists of a wearable ECG acquisition device, a mobile phone platform, and a healthcare server. Here, novel dry foam electrodes, developed to provide good conductivity without conduction gels to acquire ECG signal effectively, was used to embed into the wearable ECG acquisition device. Under the consideration of long-term ECG monitoring, the ECG acquisition module was also designed as a wireless module with low power consumption, and can be embedded into the wearable ECG acquisition device. The use of the wearable ECG acquisition device can provide more comfort and convenience for long-term ECG monitoring in daily life. Finally, this system was validated and tested to detect atrial fibrillation (AF) in Chi Mei Medical Center, Taiwan. The rest of the paper was organized as follows. Sections II and III introduced the system architecture and the system software design, respectively. In Section IV, the performance of dry foam electrode was tested and validated. The conclusion was drawn in Section V.

\section{System ARChitectURE AND DESIGN}

The system scheme of WMEMS is illustrated in Fig. 1. Here, the wearable ECG acquisition device, which consists of dry foam electrodes, an ECG acquisition module, and a wearable ECG vest, will first acquire the user's ECG signal and transmit it to the mobile phone platform via Bluetooth. The received ECG signal will be monitored continuously by an ECG monitoring MIDlet program built in the mobile phone platform. The program will also transmit average heart rate (HR) to healthcare server via SMS message every $2 \mathrm{~h}$. When the abnormal HR state occurs, the program will send a SMS alert message to the healthcare server and physicians to provide emergency treatment. Here, the short message service (SMS) alert message contains the user's raw ECG and global positioning system (GPS) information. The occurrence of broken lead, poor electrode-skin contact, or other problems may cause judgment fault of the HR detection algorithm. Physicians can then understand the actual state of patients by using the raw ECG information. This can also effectively saves the cost of telemedicine and reduces the workload of physicians caused by judgment fault.

\section{A. Design of Novel Dry Foam Electrode}

A novel dry foam electrode, as shown in Fig. 2, was designed to embed into the wearable ECG acquisition device. An electrically polymer conductive foam with the compression set about $5-10 \%$, made by urethane material, was used to contact the skin. A $0.2 \mathrm{~mm}$-thick taffeta material made electrically polymer conductive fabric (conductive about $0.07 \Omega$ /squares)

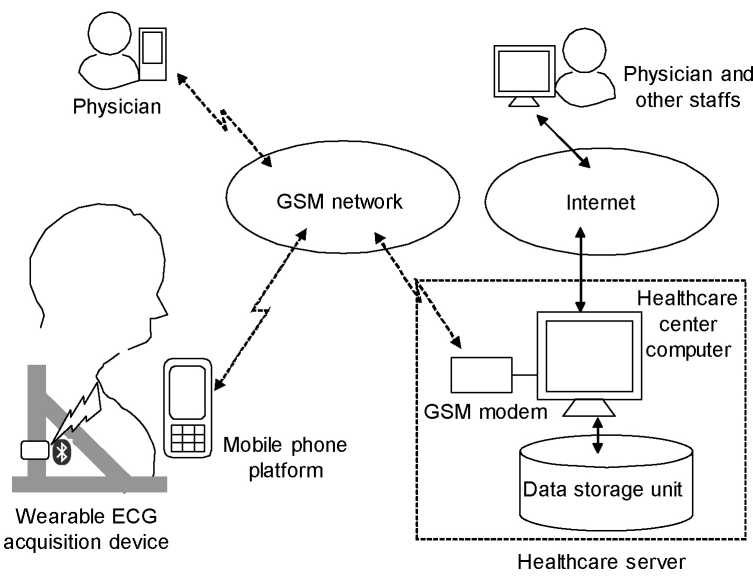

Fig. 1. Basic scheme of proposed WMEMS system.

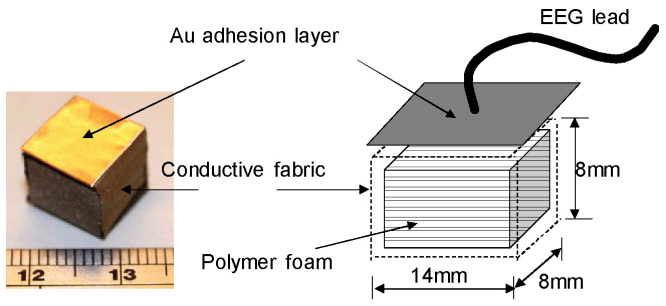

(a)

(b)

Fig. 2. (a) Top view and (b) exploded view of proposed dry foam ECG electrode.

was used to cover the electrically polymer conductive foam, and the foam was also coated with $\mathrm{Ni} / \mathrm{Cu}$ to establish an electrical contact, which can provide similar property of dry silver electrodes. And the adhesion layer is made by a $0.2 \mathrm{~nm} \mathrm{Au}$ layer. The size of the novel dry foam electrode is about 14(L) $\mathrm{mm} \times 8(\mathrm{~W}) \mathrm{mm} \times 8(\mathrm{H}) \mathrm{mm}$. The skin-electrode impedance should be directly related to the skin-electrode contact area and the skin properties of each subject. The skin-electrode impedance will be reduced as the contact area increases. By applying a suitable pressure, the dry foam electrode can adapt to the skin topography to maintain a lower skin-electrode interface impedance, and reduce the motion artifact due to its flexibility and cushioning effect.

\section{B. ECG Acquisition Module}

The ECG acquisition module is designed as a three-lead ECG device, and its system block diagram is shown in Fig. 3. It mainly consists of a front-end amplifier (FEA) circuit, an analog-to-digital converter (ADC), a microprocessor, a driven right leg circuit (DRL), and a wireless transmission circuit. The FEA circuit was designed to amplify and filter ECG signal. It contains a pre-amplifier and a band-pass filter, and its gain was set to about 112 times with frequency band of $0.05-$ $150 \mathrm{~Hz}$. The microprocessor is designed to control the ADC to acquire ECG signal, and pre-process and send ECG data to the wireless transmission circuit. Here, a 12-bit ADC was used to digitize ECG signal with the sampling rate of $512 \mathrm{~Hz}$. Here, the wireless transmission circuit consists of a Bluetooth module and a printed circuit board (PCB) antenna. The Bluetooth 


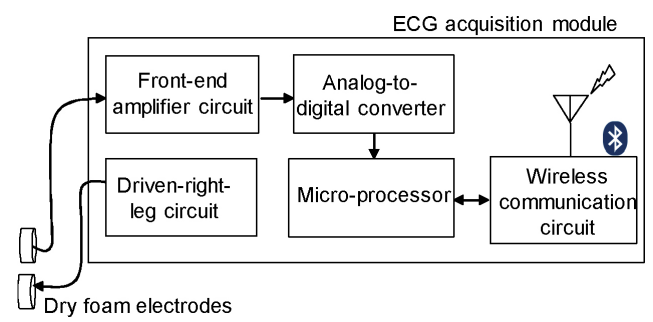

Fig. 3. Block diagram of proposed ECG acquisition module.

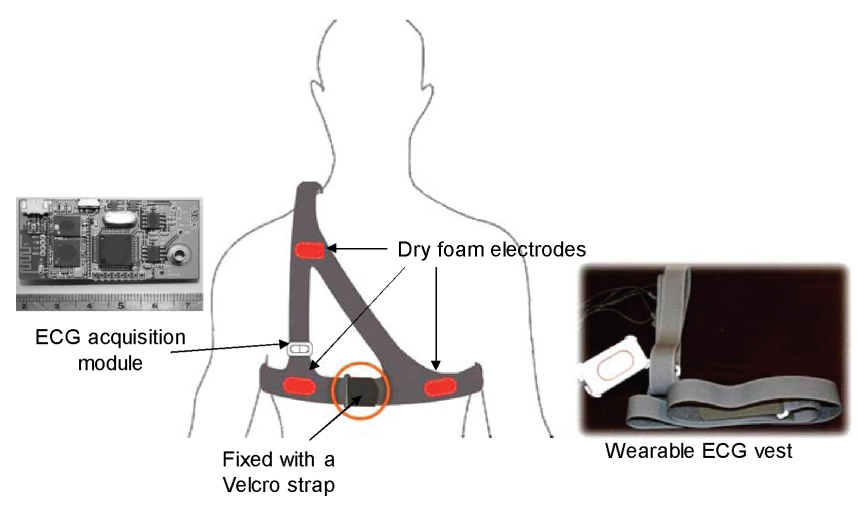

Fig. 4. Mechanical design of proposed medical vest.

module is fully compliant with the Bluetooth v2.0+EDR specification. The size of the ECG acquisition module is about $4 \mathrm{~cm} \times 2.5 \mathrm{~cm} \times 0.6 \mathrm{~cm}$, and this module operates at $31 \mathrm{~mA}$ with 3.7-V DC power supply. By using a commercial 1100 $\mathrm{mAh} \mathrm{Li-ion} \mathrm{battery,} \mathrm{its} \mathrm{operation} \mathrm{time} \mathrm{is} \mathrm{over} 33 \mathrm{~h}$.

\section{Mechanical Design of Wearable ECG Vest}

The overview of the wearable ECG vest is shown in Fig. 4. It mainly consists of three fixed coupler for dry foam electrodes, conventional three-lead standard placements, and the case of the ECG acquisition module. The vest itself is made of very elastic fabric to ensure that the dry foam electrode can provide a good contact with the user's skin. The dry foam electrodes are placed at the locations of ECG Lead II, and embedded into the vest and fixed by using the fixed coupler. The wearable ECG vest can adapt the elasticity via a Velcro strap. And the ECG acquisition module can be embedded into the small case in the vest.

\section{Mobile Phone Platform}

In this study, a commercial mobile phone is used as the system platform of monitoring ECG signal. The ECG monitoring MIDlet program built in the mobile phone platform was developed on Borland JBuilder2005 conjunction with wireless development kit 2.2, and was designed to monitor the user's HR variation. When the occurrence of abnormal HR state is detected, the program will transmit a SMS alert message to the back-end the healthcare server and physicians to provide the information for emergency treatment.

\section{E. Healthcare Server}

The healthcare server mainly consists of a GSM modem, healthcare center computer, and data storage unit. Here, the

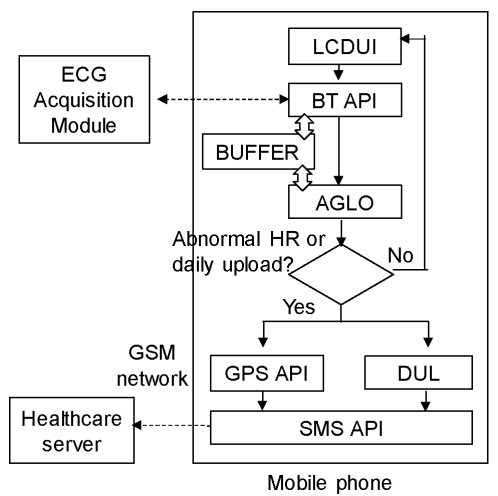

Fig. 5. Flowchart of ECG monitoring MIDlet program.

operation system of the healthcare center computer is Windows XP. The server program built in the healthcare center computer was developed on J2SE and JDesktop Integration Components (JDIC), and was designed to control the GSM modem, which is used to receive and forward SMS alert messages, by using AT-command, and to display raw ECG, HR, and GPS information.

\section{SySTEM SOFTWARE DESIGN}

\section{A. ECG Monitoring MIDlet Program in Mobile Phone}

Fig. 5 shows the flowchart of the ECG Monitoring MIDlet program. In the beginning, the program will call BT API, which is one of Bluetooth application packages used to set connection between the ECG acquisition module and the mobile phone platform, to inquiry the Bluetooth device around here. Next, BT API will try to create serial port profile (SPP) to connect the mobile phone platform to the ECG acquisition module after finding out the ECG acquisition module. After receiving raw ECG data, these ECG data will be displayed in the screen and be stored into BUFFER, which is a link-list container. Next, the thread of AGLO will detect the R-wave in ECG, and calculate the user's HR from 10-s ECG data every $5 \mathrm{~s}$. After collecting 120 trials of average HR data, the thread of DUL will package these average HR data into a daily HR message, and send it to the healthcare server every $2 \mathrm{~h}$. When the occurrence of abnormal ECG state is detected, the program will call GPS API to obtain the GPS information, and select the part of abnormal ECG signal to SMS API to send an alert message to the healthcare server.

A typical ECG waveform in AF presents a rapid irregular tachycardia, and the recognizable $\mathrm{P}$ waves are sometimes absent [18]. The range of the ventricular rate for patients with untreated AF is generally $110-180$ beats per minute, however, the ventricular rate for elderly patients with untreated $\mathrm{AF}$ is usually slower. Therefore, the irregular rhythm of the QRS complexes is usually used as the major feature of AF. The $\mathrm{R}-\mathrm{R}$ Interval (RRI), defined as the interval of neighboring QRS complexes, can reflect the information of HR, and can also be used as a parameter for identifying AF. Here, a first derivative approach (FDI), proposed in [8] and [19]-[21], was was used to detect QRS complex wave in this study. The rule for AF detection was described as follows: In the beginning, 


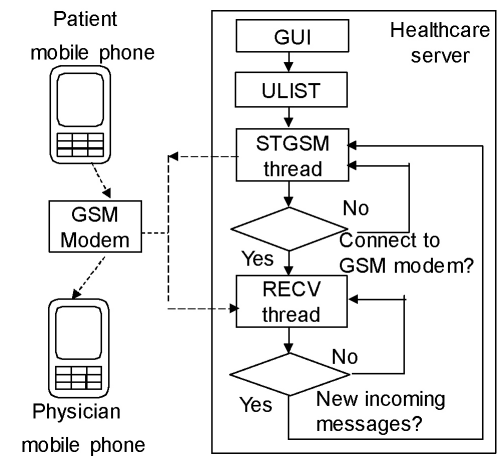

Fig. 6. Flowchart of server program in healthcare server.

the FDI approach is used to mark the R wave. Next, RRI can be calculated from the duration of adjoined $\mathrm{R}$ waves. Then the variation $\triangle R R I$ of consecutive RRI, and the standard deviation of RRI (RRIstd) within each 6-s ECG can also be calculated. The state can be viewed as AF when twice of $\triangle R R I>150$ ms occurred and RRIstd $>60 \mathrm{~ms}$ within $6 \mathrm{~s}$.

\section{B. Server Program in Healthcare Server}

The flowchart of the server program built in the healthcare center computer is shown in Fig. 6. The thread of GUI and ULIST in the program will be called to create graphic user interface when the server program begins. Next, the program will call STGSM thread to link to GSM modem. The thread of STGSM will then send an AT command to check the connection between the healthcare center computer and the GSM modem. If the connection is established, the program will create the thread of RECV to listen and wait new incoming SMS messages. The RECV thread will call STGSM to handle the new message and the program starts to extract the content in the new message, including the users' phone number, message time, SMSC number, message body, etc., when a new message is coming. After handling the new message, The RECV thread will go back to listen new incoming messages. Here, the raw abnormal ECG data and GPS information are attached in the message body.

\section{RESULTS}

\section{A. Performance Evaluation of Dry Foam Electrodes}

1) Signal-Quality Check: In order evaluate the performance of the dry foam electrode for ECG measurement, the signal quality check was examined to understand distortion caused by the dry foam electrode under ECG measurement. Fig. 7(a) illustrates the experiment for signal-quality check. First, we used the conventional wet ECG electrodes to prerecord several ECG data. And these pre-recorded ECG data was fed into a programmable function generator and a voltage divider to generate the simulated human ECG signal. Next, the ECG device with the dry foam electrodes was used to measure these simulated ECG signal. Finally, the correlation between the ECG signal measured by the dry foam electrode and the pre-recorded ECG data was calculated. The comparison between the pre-recorded ECG signal and its counterpart

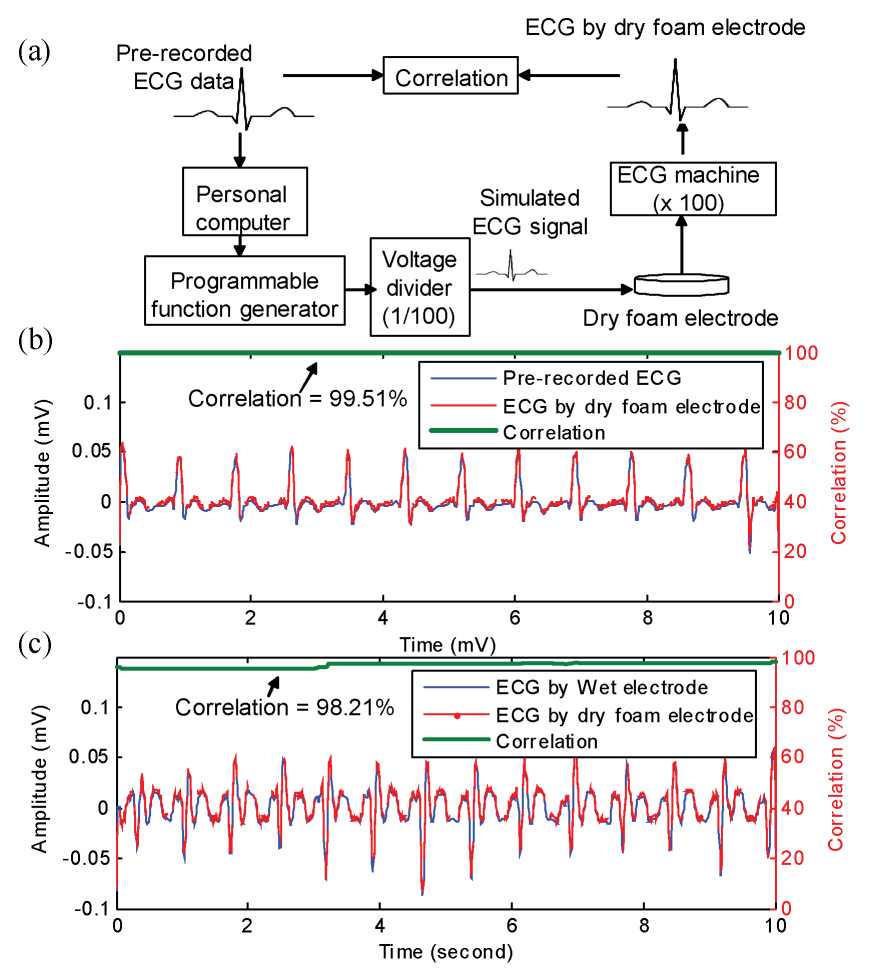

Fig. 7. (a) Experiment design for validating dry foam electrode, (b) comparison between pre-recorded ECG and ECG obtained by dry foam electrodes, and (c) comparison between ECG obtained by wet and dry foam electrodes.

recorded by the dry foam electrode is shown in Fig. 7(b), and their correlation is about $99.51 \%$. Next, the ECG signal quality of the dry foam electrode is compared with that of the conventional wet electrode. The comparison between ECG signals obtained by using the dry foam electrode and wet electrode pairs in the locations of standard Lead II sites is shown in Fig. 7(c), and their correlation is about $98.21 \%$.

2) Impedance Measurement: The electrode-skin interface impedance was analyzed by impedance spectroscopy (LCR4235, Wayne Kerr Electronics Ltd., UK) in this study, and the test signal of the impedance spectroscopy was set to $1 \mathrm{~V}$ and the frequency range from $1 \mathrm{~Hz}$ to $10 \mathrm{KHz}$. In this experiment, the conventional wet electrodes were attached to the skin using their self-adhesive properties. The dry foam electrodes were attached with a disposable Velcro strap. Both of them were exchanged carefully for each measurement to avoid the change of the skin surface state. The impedance of the skin-electrode interface corresponding to different frequencies is shown in Fig. 8(a). From the experimental result, it indicates that the impedance of the skin-electrode interface for the dry foam electrode without skin preparation and conducting gel is similar to that of the conventional wet electrode with skin preparation and conduction gel, and is prior to that of the wet electrode without skin preparation. Obviously, the conduction performance of the dry foam electrode outperforms that of the wet electrode.

Next, the impedance variation of the skin-electrode interface for long-term measurement was also investigated. Fig. 8(b) shows the courses of the impedance variations for the skin-electrode interface of the wet and dry foam electrodes. In 


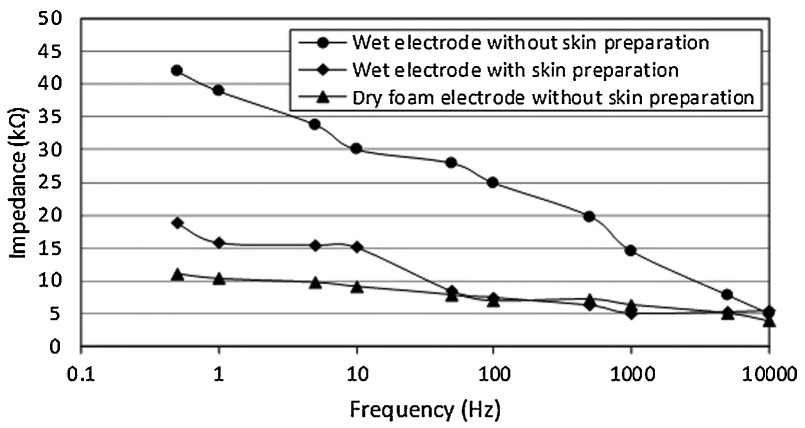

(a)

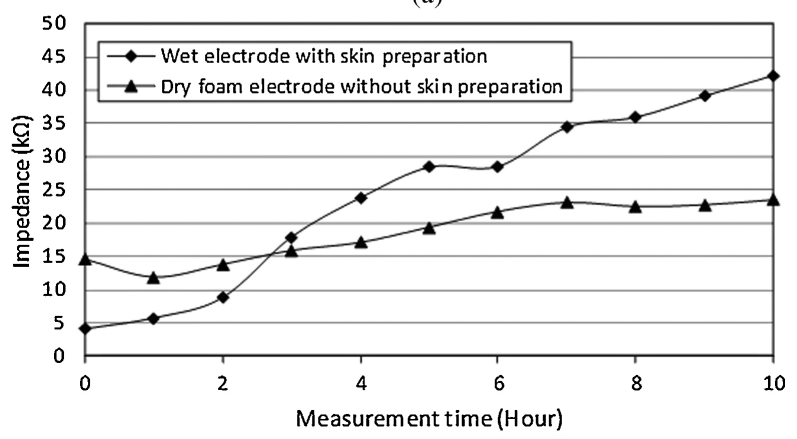

(b)

Fig. 8. (a) Frequency characteristic of proposed dry foam electrodes and (b) impedance variation of dry foam electrode and wet electrode under long-term ECG measurement.

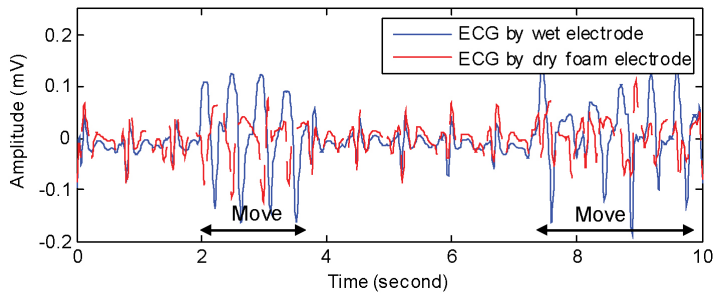

Fig. 9. Influence of motion artifact on ECG measurement of proposed dry foam and wet electrodes in a typically Lead II positions.

the first $2 \mathrm{~h}$, the impedance variation of the conventional wet electrode with conduction gel is obviously lower than that of the dry foam electrode. After two hours, the skin-electrode interface impedance of the dry foam electrode becomes close to that of the wet electrode, and even lower. The range of the impedance variation for the dry foam electrode is from 14 to $24 \mathrm{k} \Omega$, and is acceptable for ECG measurement [22]. Furthermore, for long-term measurement, the dry foam electrode can provide better stability of the skin-electrode impedance.

3) Influence of Motion Artifacts: In this section, the experiment of walking motion was performed to evaluate the influence of motion on ECG measurement. In this experiment, we instructed the participant to increase the intensity of the walking motion until the measured ECG signal was affected. The experimental result, as shown in Fig. 9, presents that the motion artifact of the conventional wet electrode is more significant than that of the dry foam electrode during the movement, especially at 2-4 and 7.6-9.7 s.

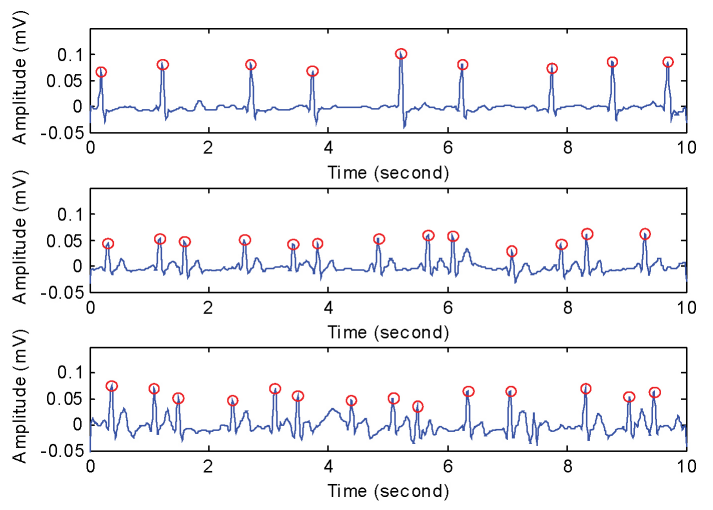

Fig. 10. Three randomly selected results of HR detection for AF.

\section{B. Accuracy Evaluation for Heart Rate Detection and Atrial Fibrillation}

In order to evaluate the accuracy of FDI approach for Rwave detection, 30-set ECG records in MIT-BIH database [23] was first used to test. The abnormal HR cases are arrhythmia and tachycardia atria fibrillation, and the normal cases are arranged in a third class. In order to check if the FDI approach can accurately detect R-wave in ECG waveform, we first defined some parameters of binary classification test. True positive (TP) indicates R-wave in ECG correctly detected as $\mathrm{R}$-wave. False positive (FP) indicates not R-wave wrongly identified as R-wave. True negative (TN) indicates not Rwave correctly identified as nothing. And False negative (FN) indicates R-wave wrongly identified as nothing. In information retrieval, the positive predictive value (PPV) and sensitivity denote the accuracy and recall, respectively. The average accuracy and sensitivity of the FDI approach for R-wave detection are 98.14 and $97.32 \%$, respectively. Therefore, the Rwave in ECG for arrhythmia and tachycardia atria fibrillation cases can exactly be detected by the FDI approach.

Next, a total of 20 AF patients (from 22 to 39 years old) participated in the clinical study, and the experiment design was performed according to the recommendations of the American National Standard for ambulatory ECG analyzers (ANSI/AAMI EC38-1994) [22]. Fig. 10 shows three randomly selected results of R-wave detection for AF cases. It shows that most of R-wave (the circle signs) for AF cases can be detected effectively. Next, we defined the parameters of binary classification test for AF detection: $\mathrm{TP}$ indicates $\mathrm{AF}$ state correctly detected as AF state. FP indicates not AF state wrongly identified as $\mathrm{AF}$ state. TN indicates not $\mathrm{AF}$ state correctly identified as nothing. And FN indicates AF state wrongly identified as nothing. The testing result is shown in Table I. The sensitivity and PPV for AF patients were 97.34 and $98.46 \%$.

\section{DISCUSSIONS}

From the results of comparing ECG signal quality obtained by dry foam electrode with the simulated ECG signal and that of the conventional wet electrode, their correlations are high. Therefore, it confirms the cleanness of our dry foam 
TABLE I

Performance of Detecting R-Wave for af CASES

\begin{tabular}{ccccc}
\hline & & & Test & \\
\multicolumn{2}{c}{ AF cases } & + & - & Total \\
\hline Truth & + & $767(\mathrm{TP})$ & $21(\mathrm{FN})$ & 788 \\
& - & $12(\mathrm{FP})$ & $0(\mathrm{TN})$ & 12 \\
\hline & Total & 779 & 21 & 800 \\
\hline
\end{tabular}

electrode from artifacts, and the signal quality of the dry foam electrodes is almost identical to that of the conventional wet electrodes. The result of long-term ECG monitoring shows that the skin-electrode impedance of the wet electrodes trend to become high over time, but that of the dry foam electrodes can effectively maintain stable skin-electrode impedance. This can be explained by that our dry foam electrode does not need conduction gel, which is apt to drying. For motion artifact, the conventional wet electrode presented more artifact than the dry foam electrode during the movement, though this may be dependent on the geometry of the dry foam electrode housing. By applying a little pressure on the dry foam electrode, the elasticity of the dry foam electrode will stabilize the skin-electrode interface both horizontally and vertically. Even if the walking motion occurs, only parts of the dry foam electrode move. Moreover, the wet electrodes suffered from moving charge artifact more than the dry foam electrodes [24]. Therefore, optimizing the application mechanism can further improve the performance of the dry foam electrode for ECG measurement. Finally, the experimental results for $\mathrm{R}$-wave detection is also significant.

The use of the proposed novel dry foam electrode provides following major merits. It is applied without conduction gels, the soft substrate of the dry foam electrode is able to adapt to irregular skin surface to maintain low skin--electrode interface impedance, and its fabrication process is low-cost. Different from MEMS electrodes, which need to penetrate into the skin, the dry foam electrode can adapt to the skin topography to guarantee small relative motion of the skin to electrode due to its flexibility and cushioning effect. However, this is also exhibits how to provide a suitable pressure to ensure the close contact between the skin and the electrode is necessary and important. Therefore, the mechanical design, which provide a suitable pressure on the dry foam electrode to maintain lower skin-electrode interface impedance, is necessarily required. Moreover, some issues for long-term ECG monitoring in daily life have also to be investigated in the future research, such as the influence of sweat and more complex actions.

\section{CONCLUSIONS}

A WMEMS was designed and implemented for long-term ECG monitoring in this study. The novel dry foam electrode was also proposed and embedded into the wearable ECG acquisition device to measure the user's ECG signal without condition gel. Experiment results show that ECG measurement by using the dry foam electrode is almost identical to that of the conventional wet electrodes with conduction gel. Moreover, for long-term ECG measurement, the dry foam electrode is more stable than the wet electrode. The property of adapting the skin for the dry foam electrode can also stabilize the skinelectrode interface, even under motion. The signal quality of the dry foam electrode was consistent for all subjects, and the variation of signal quality is very stable. Therefore, compared to the standard wet electrodes, the proposed dry foam electrode provides a potential for routine and repetitive measurement. By using the wearable ECG acquisition device, the user can monitor his/her ECG states more comfortably and conveniently in daily life. The proposed WMEMS was also tested and validated for $\mathrm{AF}$, and can effectively detect the R-wave in ECG for AF cases. Therefore, WMEMS really provides a good system prototype for telemedicine applications.

\section{REFERENCES}

[1] World Health Organization, Life in the 21st Century—A Vision for all World Health Report. Geneva, Switzerland: World Health Organization, 1998.

[2] B. S. Lin, B. S. Lin, N. K. Chou, F. C. Chong, and S. J. Chen, "RTWPMS: A real-time wireless physiological monitoring system," IEEE Trans. Inform. Technol. Biomed., vol. 10, no. 4, pp. 647-656, 2006.

[3] S. L. Chen, H. Y. Lee, C. A. Chen, H. Y. Huang, and C. H. Luo, "Wireless body sensor network with adaptive low-power design for biometrics and healthcare applications," IEEE Syst. J., vol. 3, no. 4, pp. 398-409, 2009.

[4] S. Liu, "Employing system of systems engineering in China's emergency management," IEEE Syst. J., vol. 5, no. 5, pp. 298-308, 2010.

[5] L. Duan, W. N. Street, and E. Xu, "Healthcare information systems: Data mining methods in the creation of a clinical recommender system," Enterprise Inform. Syst., vol. 5, no. 2, pp. 169-181, 2011.

[6] E. Xu, M. Wermus, and D. Bauman, "Development of an integrated medical supply information system," Enterprise Inform. Syst., vol. 5, no. 3, pp. 385-399, 2011.

[7] L. Li, L. Xu, H. A. Jeng, D. Naik, T. Allen, and M. Frontini, "Creation of environmental health information system for public health service: A pilot study," Inform. Syst. Front., vol. 10, pp. 531-543, 2008.

[8] R. G. Lee, I. C. Chou, C. C. Lai, M. H. Liu, and M. J. Chiu, "A novel QRS detection algorithm applied to the analysis for heart rate variability of patients with sleep apnea," Biomed. Eng.-Appl., Basis Commun., vol. 17, no. 5, pp. 258-262, 2005.

[9] J. E. P. Waktare, "Atrial fibrillation." Circulation, vol. 106, pp. 14-16, 2002.

[10] K. D. Bailey, "Fifty years of systems science: Further reflections," Syst. Res. Behav. Sci., vol. 22, no. 5, pp. 355-361, 2005.

[11] L. D. Xu, "The contributions of systems science to information systems research," Syst. Res. Behav. Sci., vol. 17, no. 1, pp. 105-116, 2000.

[12] G. D. Snooks, "A general theory of complex living systems: Exploring the demand side of dynamics," Complexity, vol. 13, no. 6, pp. 12-20, Jul. 2008.

[13] John N. Warfield, "A proposal for systems science," Syst. Res. Behav. Sci., vol. 20, no. 6, pp. 507-520, 2003.

[14] C. T. Lin, L. W. Ko, J. C. Chiou, J. R. Duann, R. S. Huang, T. W. Chiu, S. F. Liang, and T. P. Jung, "Noninvasive neural prostheses using mobile and wireless EEG," Proc. IEEE, vol. 96, no. 7, pp. 1167-1183, 2008.

[15] P. Griss, P. Enoksson, H. K. Tolvanen-Laakso, P. Meriläinen, S. Ollmar, and G. Stemme, "Micromachined electrodes for biopotential measurement," IEEE J. Microelectromech. Syst., vol. 10, pp. 10-15, 2001.

[16] P. Griss, H. K. Tolvanen-Laakso, P. Meriläinen, and G. Stemme, "Characterization of micromachined spiked biopotentials electrodes," IEEE Trans. Biomed. Eng., vol. 49, no. 6, pp. 597-604, 2002.

[17] C. Fonseca, J. S. Cunha, R. Martins, V. Ferreira, J. M. d. Sá, M. Barbosa, and A. M. d. Silva, "A novel dry active electrode for EEG recording," IEEE Trans. Biomed. Eng., vol. 54, no. 4, pp. 162-165, 2007.

[18] J. Hurst, "Naming of the waves in the ECG, with a brief account of their Genesis," Circulation, vol. 98, no. 18, pp. 1937-1942, 1998.

[19] G. M. Friesen, T. C. Jannett, M. A. Jadallah, S. L. Yates, S. R. Quint, and H. T. Nagle, "A comparison of the noise sensitivity of nine QRS detection algorithms," IEEE Trans. Biomed. Eng., vol. 37, no. 1, pp. 85-98, Jan. 1990. 
[20] P. Jiapu and W. J. Tompkins, "A real-time QRS detection algorithm," IEEE Trans. Biomed. Eng., vol. 32, pp. 230-236, 1985.

[21] H. L. Chan, W. S. Chou, S. W. Chen, S. C. Fang, C. S. Liou, and Y. S. Hwang, "Continuous and online analysis of heart rate variability," $J$. Med. Eng. Technol., vol. 29, no. 5, pp. 227-234, 2005.

[22] American National Standard for Ambulatory Electrocardiographs, ANSI/AAMI EC38-1994, Association for the Advancement of Medical Instrumentation, 1994.

[23] MIT-BIH Database Distribution [Online]. Available: http://ecg.mit.edu/

[24] A. Searle and J. Kirkup, "A direct comparison of wet, dry and insulating biolelectric recording electrodes," Physiol. Meas., vol. 21, pp. 271-283, 2000.

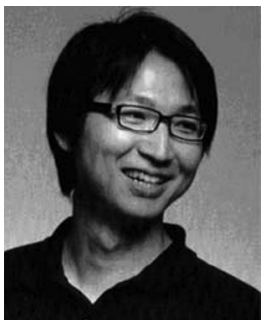

Kevin C. Tseng (M'02) is currently the Director of the Design Center and the Founder of the Product Design and Development Research Group, Chang Gung University, Taoyuan, Taiwan, which has a high reputation, both on a national and international level. It has strong links with several industries, such as Advantech Company, Ltd., Hitachi Taiwan Company, Ltd., and ITRI. He has been involved in management, consultancy, and joint projects with leading organizations in Taiwan and overseas.

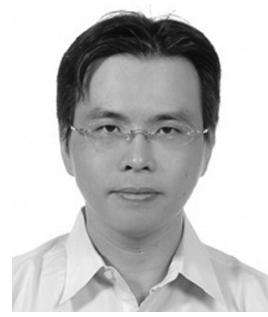

Bor-Shyh Lin (M'02) received the B.S. degree from National Chiao Tung University (NCTU), Hsinchu, Taiwan, in 1997, and the M.S. and Ph.D. degrees from National Taiwan University (NTU), Taipei, Taiwan, in 1999 and 2006, respectively, both in electrical engineering.

Currently, he is an Assistant Professor at the Institute of Imaging and Biomedical Photonics, NCTU, Hsinchu, Taiwan. His current research interests include the biomedical circuits and systems and biomedical signal processing.

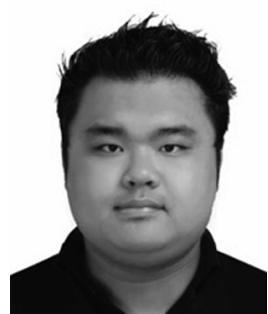

Lun-De Liao (S'08-M'10) received the B.S. degree from Chung Yuan Christian University, Jhongli, Taiwan, in 2006, the M.S. degree from National Cheng Kung University, Tainan, Taiwan, in 2008, and the Ph.D. degree from National Chiao Tung University, Hsinchu, Taiwan, in 2012.

His current research interests include biophotonic functional imaging, advanced sensing techniques, ultrafast laser systems, and relativistic molecular dynamics.

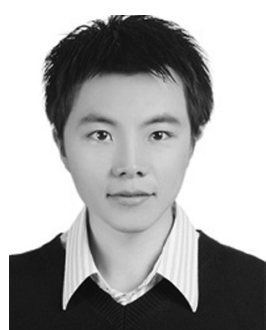

Yu-Te Wang received the M.S. degree from the Department of Biomedical Engineering, National Chiao Tung University, Taiwan, in 2009.

$\mathrm{He}$ is currently a Research Associate at the Swartz Center for Computational Neuroscience, University of California San Diego, San Diego, CA, USA. His current research interests include brain computer interface design and embedded systems.

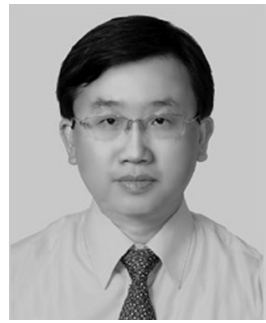

Yu-Lin Wang received the M.D. degree from Kaohsiung Medical University, Kaohsiung, Taiwan, in 1992.

Since 1996, he has been a Visiting Staff and Lecturer at the Rehabilitation Department, Kaohsiung Medical College Hospital and Chimei Medical Center, Tainan, Taiwan. His current research interests include sonograms image processing and electrophysiological signal processing. 